\title{
A NEW CHARACTERIZATION OF AMENABLE GROUPS ${ }^{1}$ \\ BY \\ JON SHERMAN
}

\begin{abstract}
Paradoxical sets, which are a natural generalization of the type of sets made famous as Hausdorff-Banach-Tarski paradoxes, are defined in terms of piecewise translations. Piecewise translations are the generalization to arbitrary discrete groups of the maps used in the Banach-Tarski paradoxes as congruences by finite decomposition. A subset of a group is defined to be large if finitely many translates of it can cover the group. The main result of this paper is that a group is amenable if and only if it does not contain a large paradoxical set.
\end{abstract}

1. Introduction. A Banach measure on a group $G$ is a finitely additive, nonnegative, translation invariant measure which is defined for every subset of $G$. A group on which a Banach measure can be defined is called amenable.

Stefan Banach [1] showed that $\mathbf{R}$, the additive group of real numbers, is amenable. von Neumann [2] observed that the same proof shows that any Abelian group is amenable. Hausdorff [3] constructed subsets of the 2-sphere which are simultaneously one-half the sphere and one-third the sphere. Starting from this paradox, Banach and Tarski [4] showed that any two bounded subsets of $E^{3}$ with nonempty interiors are congruent by finite decomposition. Two sets $A$ and $B$ are defined to be congruent by finite decomposition if each can be written as the disjoint union of $n$ subsets such that the $i$ th subset of $A$ is congruent to the $i$ th subset of $B$ for $i=1,2, \ldots, n$. It is clear that if $A$ and $B$ are subsets of an amenable group $G$ and are congruent by finite decomposition, then the measures of $A$ and $B$ are equal.

Thus Banach and Tarski showed that a Banach measure cannot be defined on the group of isometries of $E^{3}$. von Neumann [2] pointed out that these odd sets constructed by Hausdorff, Banach, and Tarski depend on the existence in the group of isometries of $E^{3}$ of a free subgroup on two generators. The natural question then is: if a group $G$ does not contain a free subgroup on two generators, is $G$ amenable? This question is yet unanswered. Another question one might ask is: does every group which fails to be amenable

Received by the editors April 19, 1976 and, in revised form, August 14, 1978.

AMS (MOS) subject classifications (1970). Primary 28A65, 28A70, 43A05, 43A07; Secondary 22D40, 47A35.

Key words and phrases. Hausdorff paradox; Banach-Tarski paradox; Banach measure; finitely additive, translation-invariant measure; congruence by finite decomposition; paradoxical set; amenable group; invariant mean; Hahn-Banach extension.

${ }^{1}$ Sincere thanks to Ernst Straus for his invaluable aid. 
contain a paradoxical set of the Banach-Tarski type, or can amenability fail in some other way? We prove in this paper that if the notion of paradoxical set is suitably generalized, then a group is amenable if and only if it does not contain a "large" paradoxical set. The word large is needed here, since there are amenable groups with paradoxical sets which can consistently be assigned measure zero.

\section{Piecewise translations and paradoxical sets.}

Definition 2.1. A piecewise translation of a subset $A$ of a group $G$ is a function $f: A \rightarrow f(A)$ such that

and

$$
A=\bigcup_{i=1}^{n} A_{i}, \quad \text { where } A_{i} \cap A_{j}=\varnothing \quad \text { if } i \neq j ;
$$

$$
\begin{gathered}
f(a)=\alpha_{i} a \text { for all } a \in A_{i}, \\
\alpha_{i} A_{i} \cap \alpha_{j} A_{j}=\varnothing \quad \text { if } i \neq j .
\end{gathered}
$$

We have identified piecewise translations with translations on the left. To be completely general we should also define right piecewise translations and two-sided piecewise translations. In Definitions 2.4 and 2.8 we will define paradoxical sets in terms of piecewise translations and proceed with the proof of the main result of this paper: that if a group does not contain such a paradoxical set, then it is amenable. Since not containing a paradoxical set by left piecewise translations is a weaker hypothesis than not containing a paradoxical set by general piecewise translations (left, right, or two-sided), we prove a slightly stronger theorem as well as gain some simplicity by working only with left piecewise translations.

THEOREM 2.2. (1) A piecewise translation is a 1-to-1 function.

(2) If $f: A \rightarrow B$ is a piecewise translation, then $f^{-1}: B \rightarrow A$ is also.

(3) The composition of piecewise translations is again a piecewise translation.

(4) The relation $B=f(A)$, where $f$ is a piecewise translation, is an equivalence relation on the subsets of $G$.

(5) If $B=f(A)$, where $f$ is a piecewise translation, then $A$ and $B$ have the same measure on any Banach measure defined on $G$.

(6) If $f$ is a piecewise translation of $S$, then its restriction to a subset $T \subset S$ is a piecewise translation of $T$.

Proof. These statements are all obvious.

Definition 2.3. A set $S \subset G$ is large if there exist piecewise translations $f_{1}, f_{2}, \ldots, f_{n}$ such that

$$
G=\bigcup_{i=1}^{n} f_{i}(S)
$$


It is easy to see that this is equivalent to the existence of finitely many elements $x_{1}, x_{2}, \ldots, x_{m}$ of $G$ such that the translates $x_{i} S$ cover $G$ (i.e., $\left.G \subset \cup \cup_{i=1}^{m} x_{i} S\right)$.

It also follows easily from this definition that if $A \subset B$ and $A$ is large then $B$ is large.

Definition 2.4. A set $S \subset G$ is $n+1-$ into- $n$ if there exist piecewise translations $f_{1}, f_{2}, \ldots, f_{n+1}$ and $g_{1}, \ldots, g_{n}$ such that

$$
\bigcup_{i=1}^{n+1} f_{i}(S) \subset \bigcup_{i=1}^{n} g_{i}(S)
$$

and

$$
f_{i}(S) \cap f_{j}(S)=\varnothing \quad \text { for } i \neq j .
$$

Definition 2.5. A set $E \subset G$ is a $\Delta$-set of the set $S \subset G$ if there is a piecewise translation $f$ such that $f(S) \subset S$ and $E=S-f(S)$.

THEOREM 2.6. The following conditions are equivalent:

(1) $G$ contains an $n+1-$ into- $n$.

(2) $G$ contains a 2-into-1.

(3) $G$ contains a set $S$ and $a \Delta$-set $E$ of $S$ such that $S \subset \cup_{i=1}^{n} f_{i}(E)$, where $f_{1}, \ldots, f_{n}$ are piecewise translations.

THEOREM 2.7. The following conditions are equivalent:

(1) $G$ contains a large $n+1-$ into- $n$.

(2) $G$ contains a large 2-into-1.

(3) $G$ contains a large $\Delta$-set.

Proof of Theorems 2.6 AND 2.7. We will prove that $3 \Rightarrow 2 \Rightarrow 1 \Rightarrow 3$ for both Theorems 2.6 and 2.7.

$3 \Rightarrow 2$. Assume $E=S-f(S)$, where $f(S) \subset S$ and $S \subset \cup_{i=1}^{n} g_{i}(E)$, where $f$ and $g_{1}, g_{2}, \ldots, g_{n}$ are piecewise translations. Then $f^{2}(S) \subset f(S)$. Next let $E_{2}=f(S)-f^{2}(S)$. In this way define $E_{i}=f^{i-1}(S)-f^{i}(S)$ for $i=$ $1, \ldots, n$. Let $E_{1}=E$. Then $E_{i} \cap E_{j}=\varnothing$ if $i \neq j$, and

$$
\bigcup_{i=1}^{n} E_{i} \subset \bigcup_{i=1}^{n}\left[f^{i-1}(S)-f^{i}(S)\right] \subset S-f^{n}(S) \text {. }
$$

Since $S \subset \cup_{i=1}^{n} g_{i}(E)$ and $E_{i}=f^{i-1}\left(E_{1}\right)$, it follows that we can define a piecewise translation $h$ such that $h(S) \subset \cup_{i=1}^{n} E_{i}$. Then $f^{n}(S) \cup h(S) \subset S$ and $f^{n}(S) \cap h(S)=\varnothing$. Thus $S$ is a 2-into-1.

If $E$ is large, then, since $E \subset S, S$ is large, and thus $S$ is a large 2-into-1.

$2 \Rightarrow 1$. This is true because a 2 -into-1 is by definition an $n+1$-into- $n$.

$1 \Rightarrow 3$. Suppose that $\cup_{i=1}^{n+1} f_{i}(S) \subset \cup_{i=1}^{n} g_{i}(S)$, where $f_{i}(S) \cap f_{j}(S)=\varnothing$ if $i \neq j$ and the $f_{i}$ and $g_{i}$ are piecewise translations. 
Let $S^{\prime}=\cup_{i=1}^{n} g_{i}(S)$ and define a piecewise translation $h: S^{\prime} \rightarrow S^{\prime}$ by

$$
\begin{gathered}
h\left(g_{1}(S)\right)=f_{1} g_{1}^{-1}\left(g_{1}(S)\right)=f_{1}(S), \\
h\left(g_{2}(S)-g_{1}(S)\right)=f_{2} g_{2}^{-1}\left(g_{2}(S)-g_{1}(S)\right) \subset f_{2}(S), \\
h\left(g_{3}(S)-g_{2}(S)-g_{1}(S)\right)=f_{3} g_{3}^{-1}\left(g_{3}(S)-g_{2}(S)-g_{1}(S)\right) \subset f_{3}(S), \\
\vdots \\
h\left(g_{n}(S)-g_{n-1}(S)-\cdots-g_{1}(S)\right) \\
=f_{n} g_{n}^{-1}\left(g_{n}(S)-g_{n-1}(S)-\cdots-g_{1}(S)\right) \subset f_{n}(S) .
\end{gathered}
$$

Then

$$
h\left(S^{\prime}\right) \subset \bigcup_{i=1}^{n} f_{i}(S) \subset \bigcup_{i=1}^{n+1} f_{i}(S) \subset \bigcup_{i=1}^{n} g_{i}(S)
$$

and

$$
E=S^{\prime}-h\left(S^{\prime}\right) \supset f_{n+1}(S) .
$$

It is easy to see that $S^{\prime} \subset \cup_{i=1}^{n} g_{i} f_{n+1}^{-1}\left(f_{n+1}(S)\right)$, and since $f_{n+1}(S) \subset E$ it follows that there exist piecewise translations $e_{1}, \ldots, e_{n}$ such that $S^{\prime} \subset$ $\cup_{i=1}^{n} e_{i}(E)$. We already know that if $A$ is large and $A \subseteq B$ then $B$ is large. Thus if we are given that $S$ is large, then it follows that $E$ is large, since $f_{n+1}(S) \subset E$.

DEFINITION 2.8. We say that a group $G$ contains a large paradoxical set if for some cyclic group $C_{n}$ the direct sum $G \times C_{n}$ contains a large 2-into-1.

THEOREM 2.9. If $G$ contains a large paradoxical set, then $G$ is not amenable.

Proof. If $S$ is a large 2-into-1 in $G \times C_{n}$, then $f_{1}(S) \cup f_{2}(S) \subset g(S)$ and $f_{1}(S) \cap f_{2}(S)=\varnothing$ implies that $2 \mu(S) \leqslant \mu(S)$ for any Banach measure $\mu$. Thus $\mu(S)=0$. But since $S$ is large, $G \times C_{n} \subset \cup_{i=1}^{m} h_{i}(S)$. Thus $m \cdot \mu(S)>$ 1.

This contradiction shows that $G \times C_{n}$ is not amenable. Since it is well known that the group $A \times B$ is amenable if $A$ and $B$ are, and that $C_{n}$ is amenable, it follows that $G$ is not amenable.

\section{Definition of upper and lower measures.}

Definition 3.1. If $A, B \subset G$, then we say $m \times A \subset n \times B$ if there exist piecewise translations $f_{1}, f_{2}, \ldots, f_{m}$ and $g_{1}, \ldots, g_{n}$ in $G \times C_{s}$ for some sufficiently large $s$ such that

$$
\bigcup_{i=1}^{m} f_{i}(A) \subset \bigcup_{i=1}^{n} g_{i}(B) \subset G \times C_{s}
$$


and

$$
f_{i}(A) \cap f_{j}(A)=\varnothing \quad \text { if } i \neq j .
$$

THEOREM 3.2. If $A, B, C, \subset G$, then:

(1) If $n_{1} \times A \subset m_{1} \times B$ and $n_{2} \times A \subset m_{2} \times B$, then

$$
\left(n_{1}+n_{2}\right) \times A \subset\left(m_{1}+m_{2}\right) \times B \text {. }
$$

(2) If $n \times A \subset m \times B$, then pn $\times A \subset p m \times B$.

(3) If $n \times A \subset m \times B$ and $m \times B \subset r \times C$, then $n \times A \subset r \times C$.

(4) If $n \times A_{1} \subset m_{1} \times B$ and $n \times A_{2} \subset m_{2} \times B$, then

$$
n \times\left(A_{1} \cup A_{2}\right) \subset\left(m_{1}+m_{2}\right) \times B \text {. }
$$

(5) If $n_{1} \times A \subset m \times B_{1}$ and $n_{2} \times A \subset m \times B_{2}$ and $B_{1} \cap B_{2}=\varnothing$, then

$$
\left(n_{1}+n_{2}\right) A \subset m \times\left(B_{1} \cup B_{2}\right) \text {. }
$$

Proof. These are all obvious consequences of Definition 3.1.

Definition 3.3. If $A \subset G$ we say $A \geqslant 1 / n$ if $G \subset n \times A$. Equivalently, $A \geqslant 1 / n$ means there exist $n$ piecewise translations $f_{1}, \ldots, f_{n}$ such that $G \subset \cup \cup_{i=1}^{n} f_{i}(A)$.

Definition 3.4. If $A \subset G$ we define the set of positive numbers $L_{A}$ as follows:

$$
a \in L_{A} \text { if } a=1 / n_{1}+1 / n_{2}+\cdots+1 / n_{k}
$$

and

$$
A=\bigcup_{i=1}^{k} A_{i}, \quad \text { where } A_{i} \cap A_{j}=\varnothing \text { if } i \neq j \text { and } A_{i} \geqslant 1 / n_{i} \text {. }
$$

We call $L_{A}$ the set of lower bounds of $A$.

THEOREM 3.5. If $G$ contains no large paradoxical sets, then $L_{A}$ is bounded above and $\sup L_{A} \leqslant 1$ for every $A \subset G$.

Proof. Assume $a \in L_{A}$ for $A \subset G$ and that $a>1$. Then $a=1 / n_{1}$ $+\cdots+1 / n_{k}$ and $A=\cup{ }_{i=1}^{k} A_{i}$, where $A_{i} \geqslant 1 / n_{i}$ and $A_{i} \cap A_{j}=\varnothing$ if $i \neq j$. Then

$$
G \subset n_{i} \times A_{i} \quad \text { for } i=1,2, \ldots, k \text {. }
$$

Let $p=n_{1} \cdot n_{2} \cdots \cdot n_{k}$. Then $p a=p / n_{1}+\cdots+p / n_{k}>p$. We are going to show that if $s$ is any positive integer greater than $p a$, then $G \times C_{s}$ contains a large $n+1$-into- $n$. Therefore choose a positive integer $s>p a$. Then by hypothesis, $G \times C_{s}$ contains no large 2 -into-1. From (1) and Theorem 3.2, it follows that $p / n_{i} \times G \subset p \times A_{i}$ for $i=1,2, \ldots, k$. Thus, by Theorem 3.2, we have $\left(\sum_{i=1}^{k} p / n_{i}\right) \times G \subset p \times A$. But $\sum_{i=1}^{k} p / n_{i}=p a$. Thus $p a \times G \subset p$ $\times A$, and since $p \times A \subset p \times G$, we have $p a \times G \subset p \times G$, where $p a>p$. 
Therefore $G$ is a large $n+1$-into- $n$ in $G \times C_{s}$, and it follows from Theorem 2.7 that $G \times C_{s}$ contains a large 2-into-1. This contradicts our assumption that $G$ contains no large paradoxical set and shows that $\sup L_{A}$ $\leqslant 1$ for every $A \subset G$.

It is interesting to note that if $G$ contains a large paradoxical set, then $\sup L_{A}$ is unbounded for all large sets. To prove this, note that a group $G$ contains a large 2-into- 1 if and only if $G$ is a large 2-into-1. Then if $A$ is a large subset of $G$ and $G \times C_{n}$ is a large 2-into-1 for some $n$, then $A$ covers $G \times C_{n}$. But the same number of images of $A$ also cover $2 \times\left(G \times C_{n}\right)$ and $4 \times\left(G \times C_{n}\right)$ and $8 \times\left(G \times C_{n}\right)$, etc.

Definition 3.6. If $G$ does not contain a large paradoxical set, we define a lower measure $\underline{\mu}(A)=\sup L_{A}$ for every $A \subset G$, where $\underline{\mu}(A)=0$ if $L_{A}=\varnothing$.

THEOREM 3.7. If $G$ is a group which does not contain a large paradoxical set, then the lower measure $\mu$ satisfies the following conditions:

(1) $\mu(A) \leqslant 1$ for every $A \subset G$.

(2) $\bar{\mu}(f(A))=\mu(A)$ for every $A \subset G$ and every piecewise translation $f$.

(3) $\bar{\mu}(G)=1$.

(4) $\underline{\mu}(A \cup B) \geqslant \underline{\mu}(A)+\underline{\mu}(B)$ for $A, B \subset G$ with $A \cap B=\varnothing$.

Proof. Statement (1) is a consequence of Theorem 3.5. Since $A>1 / n$ means that $G=\cup_{i=1}^{n} f_{i}(A)$, where $f_{1}, \ldots, f_{n}$ are piecewise translations, it follows that $g(A) \geqslant 1 / n$ for any piecewise translation $g$. Thus if $a \in L_{A}$, then $a \in L_{g(A)}$. This proves (2).

To show (3) we observe that $G=G$. Thus $1 \in L_{G}$, and from Theorem 3.5 we have $\sup L_{G} \leqslant 1$. Thus $\sup L_{G}=1$.

To prove (4), suppose $\alpha \in L_{A}$ and $\beta \in L_{B}$. Then $A=\cup_{i=1}^{k} A_{i}$, where $A_{i} \geqslant 1 / n_{i}, A_{i} \cap A_{j}=\varnothing$ if $i \neq j$ and $\alpha=\sum_{i=1}^{k} 1 / n_{i}$; and $B=\cup_{i=1}^{t} B_{i}$, where $B_{i} \geqslant 1 / m_{i}$ and $B_{i} \cap B_{j}=\varnothing$ if $i \neq j$ and $\beta=\sum_{i=1}^{t} 1 / m_{i}$. Then $A \cup B$ $=\cup_{i=1}^{k} A_{i} \cup\left(\cup_{i=1}^{t} B_{i}\right)$.

Thus $\alpha+\beta \in L_{A \cup B}$ and $\sup L_{A \cup B} \geqslant \alpha+\beta$. Since $\alpha$ and $\beta$ are arbitrary elements of $L_{A}$ and $L_{B}$, it follows that

$$
\sup L_{A \cup B} \geqslant \sup L_{A}+\sup L_{B} \text {. }
$$

Definition 3.8. If $A \subset G$ we say that $A \leqslant 1 / n$ if $n \times A \subset G . A<1 / n$ is equivalent to the existence of $n$ piecewise translations $f_{1}, \ldots, f_{n}$ such that $\cup_{i=1}^{n} f_{i}(A) \subset G$ and $f_{i}(A) \cap f_{j}(A)=\varnothing$ if $i \neq j$.

Definition 3.9. If $A \subset G$ we associate with $A$ the set of positive numbers $U_{A}$, the set of upper bounds of $A: U_{A}=\left\{\alpha: \alpha=\sum_{i=1}^{k} 1 / n_{i}\right.$ and $A=\cup_{i=1}^{k} A_{i}$, where $\left.A_{i} \leqslant 1 / n_{i}\right\}$.

Definition 3.10. If $G$ is a group which does not contain a large paradoxical set, then we define an upper measure $\bar{\mu}(A)=\inf U_{A}$, for every $A \subset G$. 
THEOREM 3.11. If $G$ is a group which does not contain a large paradoxical set, then the upper measure $\bar{\mu}$ satisfies the following conditions:

(1) $\bar{\mu}(G)=1$.

(2) $\bar{\mu}(f(A))=\bar{\mu}(A)$ for every $A \subset G$ and every piecewise translation $f$.

(3) $\bar{\mu}(A \cup B) \leqslant \bar{\mu}(A)+\bar{\mu}(B)$ for $A, B \subset G$ with $A \cap B=\varnothing$.

(4) $\bar{\mu}(A) \leqslant \bar{\mu}(B)$ if $A \subset B$.

Proof. (1) Suppose there is an $\alpha \in U_{G}$ with $\alpha<1$. Then $G=\cup_{i=1}^{k} A_{i}$ with $A_{i}<1 / n_{i}$ and $\alpha=\sum_{i=1}^{k} 1 / n_{i}<1$. Thus $n_{i} \times A_{i} \subset G$ for $i=1, \ldots, k$. Let $P=n_{1} \cdot n_{2} \cdot n_{3} \cdots \cdot n_{k}$. Then, by Theorem 3.2,

$$
\left(\left(P / n_{i}\right) \cdot n_{i}\right) \times A_{i} \subset P / n_{i} \times G \text { for } i=1,2, \ldots, k \text {. }
$$

Thus

$$
P \times A_{i} \subset P / n_{i} \times G \text { for } i=1,2, \ldots, k
$$

Then

$$
P \times \bigcup_{i=1}^{k} A_{i} \subset \sum_{i=1}^{k} P / n_{i} \times G
$$

follows from Theorem 3.2.

Since $\cup_{i=1}^{k} A_{i}=G$ and $\sum_{i=1}^{k} P / n_{i}=P \alpha<P$, this implies $P \times G \subset P \alpha$ $\times G$, where $P>P \alpha$.

Thus $G$ is a large $n+1$-into- $n$ in $G \times C_{s}$ for some $s$. Then we know from Theorem 2.7 that $G \times C_{s}$ contains a large 2-into-1 and therefore $G$ contains a large paradoxical set. This contradiction shows that for every $\alpha \in U_{G}, \alpha>1$. Since $G \subset G$, we have $1 \in U_{G}$; thus inf $U_{G}=1$ and $\bar{\mu}(G)=1$.

The proof of (2) is a simple consequence of the fact that the property $A<1 / n$ is by definition invariant under piecewise translation.

To prove (3), assume that $\alpha \in U_{A}$ and $\beta \in U_{B}$. Then $A=\cup_{i=1}^{k} A_{i}$, where $A_{i} \leqslant 1 / n_{i}$ and $B=\cup_{i=1}^{t} B_{i}$, where $B_{i} \leqslant 1 / m_{i}$, and $\alpha=\sum_{i=1}^{k} 1 / n_{i}$ and $\beta=\sum_{i=1}^{t} 1 / m_{i}$. Then

$$
A \cup B=\left(\bigcup_{i=1}^{k} A_{i}\right) \cup\left(\bigcup_{i=1}^{t} B_{i}\right),
$$

where $A_{i} \leqslant 1 / n_{i}$ and $B_{i} \leqslant 1 / m_{i}$. Thus

$$
\sum_{i=1}^{k} \frac{1}{n_{i}}+\sum_{i=1}^{t} \frac{1}{m_{i}}=\alpha+\beta \in U_{A+B} .
$$

Thus inf $U_{A+B} \leqslant \alpha+\beta$. Since $\alpha$ and $\beta$ were arbitrary elements of $U_{A}$ and $U_{B}$, it follows that inf $U_{A+B} \leqslant \inf U_{A}+\inf U_{B}$.

To prove (4) we first observe that if $A_{i} \subset B_{i}$ and $B_{i} \leqslant 1 / n_{i}$, then $A_{i} \leqslant 1 / n_{i}$.

Now suppose $\alpha \in U_{B}$ and $A \subset B$. Then $B=\cup_{i=1}^{k} B_{i}$, where $B_{i}<1 / n_{i}$ 
and $\alpha=\sum_{i=1}^{k} 1 / n_{i}$. Then let $A_{i}=A \cap B_{i}$. Hence $A_{i}<1 / n_{i}$ and $A=$ $\cup{ }_{i=1}^{k} A_{i}$; therefore $\alpha \in U_{A}$, and so inf $U_{A} \leqslant \inf U_{B}$.

One may observe that if $G$ contains a large 2-into-1, then inf $U_{G}=0$.

THEOREM 3.12. If $G$ does not contain a large paradoxical set, then $\underline{\mu}(A) \leqslant$ $\bar{\mu}(A)$ for every $A \subset G$.

The point of this theorem is to show that if $G$ does not contain large paradoxical sets, then there is always room between the lower and upper measures to assign a Banach measure. The upper and lower measures of a subset of $G$ are forced by the conditions of finite additivity and translation invariance, and if there is no room between them, then amenability fails. If in fact $G$ contains a large 2-into-1, then this does fail in the wildest possible way, since $\mu(G)=\infty>\bar{\mu}(G)=0$.

Próf. Suppose $\sup L_{A}>\inf U_{A}$. Then there exist $\alpha=\sum_{i=1}^{k} 1 / n_{i} \in L_{A}$ and $\beta=\sum_{j=1}^{t} 1 / m_{i} \in U_{A}$, with $\alpha>\beta$. Then $A=\cup_{i=1}^{k} A_{i}, A_{i} \cap A_{j}=\varnothing$ if $i \neq j$; and $A=\cup_{j=1}^{t} B_{j}$ with $G \subset n_{i} \times A_{i}$ for $i=1, \ldots, k$ and $m_{i} \times B_{i} \subset$ $G$ for $i=1, \ldots, t$. Let $Q=\Pi_{i=1}^{k} n_{i}$ and $P=\prod_{j=1}^{t} m_{j}$. Then $\left(\left(Q P / m_{j}\right) \cdot m_{j}\right)$ $\times B_{j} \subset Q P / m_{j} \times G$ for $j=1, \ldots, t$; and $Q P / n_{i} \times G \subset\left(\left(Q P / n_{i}\right) \cdot n_{i}\right) \times$ $A_{i}$ for $i=1, \ldots, k$. Thus $Q P / n_{i} \times G \subset Q P \times A_{i}$ for $i=1, \ldots, k$; and $Q P \times B_{j} \subset Q P / m_{j} \times G$ for $j=1, \ldots, t$.

Thus $\left(\sum_{i=1}^{k} Q P / n_{i}\right) \times G \subset Q P \times A$ and $Q P \times A \subset\left(\sum_{j=1}^{t} Q P / m_{j}\right) \times G$. Then $Q P \alpha \times G \subset Q P \times A$ and $Q P \times A \subset Q P \beta \times G$. And so $Q P \alpha \times G \subset$ $Q P \beta \times G$. If $\alpha>\beta$, this implies that $G$ contains a large paradoxical set. Thus $\alpha \leqslant \beta$ and $\sup L_{A} \leqslant \inf U_{A}$ and $\underline{\mu}(A) \leqslant \bar{\mu}(A)$.

\section{Rational sets.}

Definition 4.1. If $G$ is a group with no large paradoxical sets, then a subset $A \subset G$ is a rational set if $\mu(A)=\bar{\mu}(A)$.

For rational sets $A$ we set $\mu(A)=\underline{\mu}(A)=\bar{\mu}(A)$.

THeOREM 4.2. If $A$ and $B$ are rational subsets of a group $G$ with no large paradoxical set and $A \cap B=\varnothing$, then $A \cup B$ is a rational set and $\mu(A \cup B)=$ $\mu(A)+\mu(B)$.

Proof. $\mu(A \cup B) \geqslant \mu(A)+\mu(B)=\bar{\mu}(A)+\bar{\mu}(B) \geqslant \bar{\mu}(A \cup B)$. Thus $\mu(A$ $\cup B) \geqslant \bar{\mu}(A \cup B)$. But $\mu(A \cup \bar{B}) \leqslant \bar{\mu}(A \cup B)$. Therefore $\mu(A \cup B)=\overline{\bar{\mu}}(A$ $\cup B)$ and $A \cup B$ is a rational set with $\mu(A \cup B)=\mu(A)+\mu(B)$.

THEOREM 4.3. If $A$ is a rational set, then so is $f(A)$ for any piecewise translation $f$, and $\mu(f(A))=\mu(A)$.

Proof. This follows from Theorems 3.11 and 3.7. 
THEOREM 4.4. On the collection of rational subsets of $G$, where $G$ contains no large paradoxical set, the set function $\mu$ is finitely additive and piecewise translation invariant with $\mu(G)=1$.

Proof. This is a consequence of Theorems 3.7, 3.11, 4.2, and 4.3.

5. Rational functions and nonnegative linear functionals. We have now shown that if $G$ is a group without large paradoxical sets then we can define a finitely additive, piecewise translation invariant set function $\mu$ on the collection of rational subsets of $G$ such that $\mu(\varnothing)=0$ and $\mu(G)=1$. (It is clear that $\varnothing$ and $G$ are rational sets.) We now wish to extend this set function to a measure on every subset of $G$. For the rational sets $A \subset G$ we have $\mu(A)=$ $\bar{\mu}(A)$, and thus there is no choice but to choose $\mu(A)=\mu(A)=\bar{\mu}(A)$. However, if $A$ is not rational, then $\mu(A)<\bar{\mu}(A)$, and we have infinitely many choices for $\mu(A)$. To make these choices consistently we wish to use a variation of the Hahn-Banach extension theorem. To that end we make the following definitions:

Definition 5.1. Let $V$ be the vector space generated by characteristic functions $\chi_{A}$ of subsets $A \subset G$. Then, if $f \in V$,

$$
f=\sum_{i=1}^{n} \alpha_{i} \chi_{A_{i}},
$$

where $\alpha_{i} \in \mathbf{R}$ and $A_{i} \subset G$.

Let $M$ be the subspace of $V$ generated by the characteristic functions of rational subsets of $G$. If $f \in M$, then

$$
f=\sum_{i=1}^{n} \alpha_{i} \chi_{A_{i}}
$$

where $\alpha_{i} \in \mathbf{R}$, and $A_{i}$ are rational subsets of $G$.

Definition 5.2. We want to define a linear functional $\lambda$ on $M$ as follows. If $f \in M$ and

$$
f=\sum_{i=1}^{n} \alpha_{i} \chi_{A_{i}}
$$

where $A_{i}$ is a rational subset of $G$ for $i=1,2, \ldots, n$, then

$$
\lambda(f)=\sum_{i=1}^{n} \alpha_{i} \cdot \mu\left(A_{i}\right)
$$

We need to show that this is a proper definition, i.e., that $\sum_{i=1}^{n} \alpha_{i} \cdot \mu\left(A_{i}\right)$ is well defined. The remainder of this section will be devoted to proving that we can define a linear functional this way.

Definition 5.3. A piecewise isomorphism is a one-to-one function $\varphi$ : $G \stackrel{\text { onto }}{\rightarrow} G^{\prime}$ such that if $f$ is a piecewise translation of a subset $A \subset G$, then $f^{\prime}(x)=\varphi f \varphi^{-1}(x)$ is a piecewise translation of $\varphi(A) \subset G^{\prime}$. 
THEOREM 5.4. If $\varphi: G \rightarrow G^{\prime}$ is a piecewise isomorphism, and $G^{\prime}$ is amenable, then $G$ is amenable.

Proof. Suppose $m^{\prime}$ is a Banach measure on $G^{\prime}$. Then define $m(A)=$ $m^{\prime}(\varphi(A))$ for every $A \subset G$. Then $m$ is a Banach measure on $G$ :

(1) $m(G)=m^{\prime}(\varphi(G))=m^{\prime}\left(G^{\prime}\right)=1$.

(2) If $A \cap B=\varnothing$, then

$m(A \cup B)=m^{\prime}(\varphi(A \cup B))=m^{\prime}(\varphi(A) \cup \varphi(B))=m^{\prime}(\varphi(A))+m^{\prime}(\varphi(B))$, since $\varphi(A) \cap \varphi(B)=\varnothing$.

(3) If $f$ is a piecewise translation,

$$
m(f(A))=m^{\prime}(\varphi(f(A)))=m^{\prime}\left(f^{\prime}(\varphi(A))\right)=m^{\prime}(\varphi(A))=m(A),
$$

where $f^{\prime}=\varphi f \varphi^{-1}$ is a piecewise translation of $\varphi(A) \subset G$.

THEOREM 5.5. If $\varphi: G \rightarrow G^{\prime}$ is a piecewise isomorphism and $G$ contains a 2-into-1 (resp., large 2-into-1), then $G^{\prime}$ contains a 2-into-1 (resp., large 2into-1).

Proof. Suppose $f_{1}(A) \cup f_{2}(A) \subset A$ and $f_{1}(A) \cap f_{2}(A)=\varnothing$, where $f_{1}$ and $f_{2}$ are piecewise translations. Then $f_{1}^{\prime}=\varphi f_{1} \varphi^{-1}$ and $f_{2}^{\prime}=\varphi f_{2} \varphi^{-1}$ are piecewise translations, and

$$
f_{1}^{\prime}(\varphi(A)) \cup f_{2}^{\prime}(\varphi(A)) \subset \varphi A \quad \text { and } \quad f_{1}^{\prime}(\varphi(A)) \cap f_{2}^{\prime}(\varphi(A))=\varnothing .
$$

If $A$ is large, then $G \subset \cup \cup_{i=1}^{n} g_{i}(A)$, where the $g_{i}$ are piecewise translations. Then $G^{\prime} \subset \cup{ }_{i=1}^{n} g_{i}^{\prime}(\varphi(A))$ and the $g_{i}^{\prime}=\varphi g_{i} \varphi^{-1}$ are piecewise translations. Thus $\varphi(A)$ is large in $G^{\prime}$.

TheOREM 5.6. If $\varphi: G \rightarrow G^{\prime}$ and $\psi: H \rightarrow H^{\prime}$ are piecewise isomorphisms, then so is $\varphi \times \psi: G \times H \rightarrow G^{\prime} \times H^{\prime}$ defined by

$$
\varphi \times \psi(g \cdot h)=\varphi(g) \psi(h)
$$

for every $g h \in G \times H$, with $g \in G, h \in H$.

Proof. If $f: S \rightarrow f(S)$ is a piecewise translation on $G \times H$, then $S=$ $\bigcup_{i=1}^{n} A_{i}$ and $f(S)=\bigcup{ }_{i=1}^{n} \alpha_{i} A_{i}$. Each $\alpha_{i}=\beta_{i} \varepsilon_{i}$ where $\beta_{i} \in G$ and $\varepsilon_{i} \in H$. Then let $f^{\prime}: \varphi \times \psi(S) \rightarrow \varphi \times \psi(f(S))$ be defined by

$$
f^{\prime}(x)=\varphi \times \psi \cdot f \cdot(\varphi \times \psi)^{-1}(x)
$$

for every $x \in S^{\prime}=\varphi \times \psi(S)$. If $(a, b)=(\varphi \times \psi)^{-1}(x) \in G \times H$, then

$$
f^{\prime}(x)=\varphi \times \psi \cdot f(a, b)=\varphi \times \psi\left(\beta_{i} a, \varepsilon_{i} b\right)=\varphi\left(\beta_{i} a\right) \psi\left(\varepsilon_{i} b\right) .
$$

The 1-to-1 function $f^{\prime}(x)$ is a piecewise translation on $S^{\prime} \subset G^{\prime} \times H^{\prime}$ if and only if the set of translates $\left\{f^{\prime}(x) \cdot x^{-1}: x \in S^{\prime}\right\}$ is finite. This is just the set 


$$
\begin{aligned}
\left\{\varphi\left(\beta_{i} a\right) \psi\left(\varepsilon_{i} b\right) \cdot[\varphi \times \psi(a b)]^{-1}: a b \in S\right\} & \\
& =\left\{\varphi\left(\beta_{i} a\right)[\varphi(a)]^{-1} \psi\left(\varepsilon_{i} b\right)[\psi(b)]^{-1}: a b \in S\right\}
\end{aligned}
$$

Let

$$
\pi_{G}\left(A_{i}\right)=\left\{a \in G:(a, b) \in A_{i}\right\}
$$

and

$$
\pi_{H}\left(A_{i}\right)=\left\{b \in H:(a, b) \in A_{i}\right\} .
$$

Define a translation $f_{i}$ on $\pi_{G}\left(A_{i}\right)$ by $f_{i}(a)=\beta_{i} a$; and a translation $h_{i}$ on $\pi_{H}\left(A_{i}\right)$ by $h_{i}(b)=\varepsilon_{i} b$. Then both $\left\{\varphi f_{i}(a) \cdot[\varphi(a)]^{-1}: a \in \pi_{G}\left(A_{i}\right)\right\}$ and $\left\{\psi h_{i}(b) \cdot\right.$ $\left.[\psi(b)]^{-1}: b \in \pi_{H}\left(A_{i}\right)\right\}$ are finite or, equivalently, $\left\{\varphi\left(\beta_{i} a\right) \cdot[\varphi(a)]^{-1}: a \in\right.$ $\left.\pi_{G}\left(A_{i}\right)\right\}$ and $\left\{\psi\left(\varepsilon_{i} b\right) \cdot[\psi(b)]^{-1}: b \in \pi_{H}\left(A_{i}\right)\right\}$ are finite for $i=1,2, \ldots, n$.

Then

$$
\bigcup_{i=1}^{n}\left\{\varphi\left(\beta_{i} a\right) \cdot[\varphi(a)]^{-1}: a \in \pi_{G}\left(A_{i}\right)\right\}=\left\{\varphi\left(\beta_{i} a\right) \cdot[\varphi(a)]^{-1}: a \in \pi_{G}(S)\right\}
$$

and

$$
\bigcup_{i=1}^{n}\left\{\psi\left(\varepsilon_{i} b\right) \cdot[\psi(b)]^{-1}: b \in \pi_{H}\left(A_{i}\right)\right\}=\left\{\psi\left(\varepsilon_{i} b\right) \cdot[\psi(b)]^{-1}: b \in \pi_{H}(S)\right\}
$$

are finite. Thus

$$
\begin{aligned}
\left|\left\{\varphi\left(\beta_{i} \alpha\right)[\varphi(a)]^{-1} \psi\left(\varepsilon_{i} b\right)[\psi(b)]^{-1}: a b \in S\right\}\right| & \\
< & \left|\left\{\varphi\left(\beta_{i} a\right)[\varphi(a)]^{-1}: a \in \pi_{G}(S)\right\}\right| \\
& \cdot\left|\left\{\psi\left(\varepsilon_{i} b\right) \cdot[\psi(b)]^{-1}: b \in \pi_{H}(S)\right\}\right|<\infty .
\end{aligned}
$$

Thus $f^{\prime}$ is a piecewise translation of $\varphi \times \psi(S)$ to $\varphi \times \psi(f S)$ in $G^{\prime} \times H^{\prime}$. Since it is clear that $\varphi \times \psi$ is 1-to-1 and onto, it follows that $\varphi \times \psi$ is a piecewise isomorphism.

Corollary 5.6'. If $\varphi$ is a piecewise isomorphism from $G$ to $G^{\prime}$ and $h$ is any 1-to-1 function from the finite group $A$ onto the finite group $B$, then $\varphi_{h}$ : $G \times A \rightarrow G \times B$ defined by $\varphi_{h}(g a)=\varphi g \cdot h a$ is a piecewise isomorphism.

Proof. Since on a finite group any 1-to-1 function is a piecewise translation, it follows that any 1-to-1 function from one finite group onto another finite group is a piecewise isomorphism. Then this is just a special case of Theorem 5.6.

COROLlaRY 5.7. If $\varphi: G \rightarrow G^{\prime}$ is a piecewise isomorphism and $G$ contains a (large) paradoxical set, then $G^{\prime}$ contains a (large) paradoxical set.

Proof. This is a consequence of Theorems 5.5 and 5.6 and the definitions of paradoxical and large paradoxical sets. 
LEMMA 5.8. If $G$ is a group without large paradoxical sets, then for any positive integer $s$ we can define a piecewise translation invariant, finitely additive set function $m$ on the rational subsets of $G \times C_{s}$ such that if $A \subset G$ is a rational set in $G$, then $m(A)=\mu(A)$.

Proof. Since $G$ contains no large paradoxical set, it follows that $G \times C_{s}$ contains no large paradoxical set, because a large 2-into-1 in $G \times C_{s} \times C_{n}$ implies the existence of a large 2-into-1 in $G \times C_{s \cdot n}$. This follows from Corollary 5.6', since $C_{s} \times C_{n}$ and $C_{s \cdot n}$ are of the same order.

Then we can define a piecewise translation invariant, finitely additive set function $m^{*}$ on the rational subsets of $G \times C_{s}$. $G$ is a rational subset of $G \times C_{s}$, and if $A$ is a rational subset of $G$, it is also a rational subset of $G \times C_{s}$. Also, $m^{*}(G)=1 / s$ and $m^{*}(A)=(1 / s) \mu(A)$. Then if we define $m(A)=s \cdot m^{*}(A)$, we are done.

LEMMA 5.9. If there is an $f \in M$ such that

$$
f=\sum_{i=1}^{n} \alpha_{i} \chi_{A_{i}}=\sum_{i=1}^{m} \beta_{i} \chi_{B_{i}}
$$

with $\alpha_{i}$ and $\beta_{i} \in \mathbf{R}$, where $A_{i}$ and $B_{i}$ are rational sets, and

$$
\sum_{i=1}^{n} \alpha_{i} \mu\left(A_{i}\right) \neq \sum_{i=1}^{m} \beta_{i} \mu\left(B_{i}\right)
$$

then there is an $f^{\prime} \in M$ such that

$$
f^{\prime}=\sum_{i=1}^{n} \alpha_{i}^{\prime} \chi_{A_{i}}=\sum_{j=1}^{m} \beta_{j}^{\prime} \chi_{B_{j}}
$$

where the $\alpha_{i}^{\prime}, \beta_{j}^{\prime} \in \mathbf{Z}$, and

$$
\sum_{i=1}^{n} \alpha_{i}^{\prime} \mu\left(A_{i}\right) \neq \sum_{j=1}^{m} \beta_{j}^{\prime} \mu\left(B_{j}\right)
$$

Proof. Let $C$ be a Hamel basis of $\mathbf{R}$ over $\mathbf{Q}$. Then for each $\alpha_{i}$ and $\beta_{i}$ there is a unique function $\alpha_{i}(c), \beta_{i}(c)$ from $C \rightarrow \mathbf{Q}$ which equals 0 except for finitely many $c$ 's such that

$$
\alpha_{i}=\sum_{c \in C} \alpha_{i}(c) \cdot c \text { and } \beta_{i}=\sum_{c \in C} \beta_{i}(c) \cdot c .
$$

Then for each $x \in G$ we have

$$
f(x)=\sum_{i=1}^{n}\left(\sum_{c \in C} \alpha_{i}(c) \cdot c\right) \chi_{A_{i}}(x)=\sum_{i=1}^{m}\left(\sum_{c \in C} \beta_{i}(c) \cdot c\right) \chi_{B_{i}}(x) .
$$

Let $c_{1}, c_{2}, \ldots, c_{s}$ be the finitely many elements of $C$ such that $\alpha_{i}(c) \neq 0$ or $\beta_{i}(c) \neq 0$ for some $i$. Then 


$$
\sum_{i=1}^{n} \sum_{j=1}^{s} \alpha_{i}\left(c_{j}\right) \cdot c_{j} \cdot \chi_{A_{i}}(x)=\sum_{i=1}^{m} \sum_{j=1}^{s} \beta_{i}\left(c_{j}\right) \cdot c_{j} \cdot \chi_{B_{i}}(x)
$$

From the linear independence of $C$ it follows that

$$
\sum_{i=1}^{n} \alpha_{i}\left(c_{j}\right) \cdot \chi_{A_{i}}(x)=\sum_{i=1}^{m} \beta_{i}\left(c_{j}\right) \cdot \chi_{B_{i}}(x)
$$

Moreover, if

$$
\sum_{i=1}^{n} \alpha_{i}\left(c_{j}\right) \mu\left(A_{i}\right)=\sum_{i=1}^{m} \beta_{i}\left(c_{j}\right) \mu\left(B_{i}\right)
$$

for each $j=1, \ldots, s$, then

$$
\sum_{j=1}^{s} \sum_{i=1}^{n} \alpha_{i}\left(c_{j}\right) \cdot c_{j} \cdot \mu\left(A_{i}\right)=\sum_{j=1}^{s} \sum_{i=1}^{m} \beta_{i}\left(c_{j}\right) \cdot c_{j} \cdot \mu\left(B_{i}\right),
$$

contradicting the hypothesis of the lemma.

Therefore, for some $j$,

$$
\sum_{i=1}^{n} \alpha_{i}\left(c_{j}\right) \mu\left(A_{i}\right) \neq \sum_{i=1}^{m} \beta_{i}\left(c_{j}\right) \mu\left(B_{i}\right),
$$

while

$$
\sum_{i=1}^{n} \alpha_{i}\left(c_{j}\right) \chi_{A_{i}}=\sum_{i=1}^{m} \beta_{i}\left(c_{j}\right) \chi_{B_{i}}
$$

and the $\alpha_{i}\left(c_{j}\right)$ and $\beta_{i}\left(c_{j}\right)$ are rational. We can now multiply through by the common denominator of the $\alpha_{i}\left(c_{j}\right)$ and the $\beta_{i}\left(c_{j}\right)$ to get the desired relations with integral coefficients.

LEMMA 5.10. If $G$ is a group with no large paradoxical sets and

$$
f=\sum_{i=1}^{n} \alpha_{i} \chi_{A_{i}}=\sum_{i=1}^{m} \beta_{i} \chi_{B_{i}}
$$

is a real-valued rational function on $G\left(A_{i}, B_{i}\right.$ are rational sets) such that

$$
\sum_{i=1}^{n} \alpha_{i} \mu\left(A_{i}\right) \neq \sum_{i=1}^{m} \beta_{i} \mu\left(B_{i}\right),
$$

then there is a rational function

$$
f^{\prime}=\sum_{i=1}^{n^{\prime}} \alpha_{i}^{\prime} \chi_{A_{i}^{\prime}}=\sum_{i=1}^{m^{\prime}} \beta_{i}^{\prime} \chi_{B_{i}^{\prime}}
$$

such that

$$
\sum_{i=1}^{n^{\prime}} \alpha_{i}^{\prime} \mu\left(A_{i}^{\prime}\right) \neq \sum_{i=1}^{m^{\prime}} \beta_{i}^{\prime} \mu\left(B_{i}^{\prime}\right)
$$

and the $\alpha_{i}$ and $\beta_{i}$ are positive integers. 
Proof. According to the preceding lemma, we may assume that the $\alpha_{i}$ and $\beta_{i}$ are integers. Suppose $\alpha_{i}, i=r+1, \ldots, n$, and $\beta_{i}, i=s+1, \ldots, m$, are negative. Define

$$
f^{\prime}=\sum_{i=1}^{r} \alpha_{i} \chi_{A_{i}}-\sum_{i=s+1}^{m} \beta_{i} \chi_{B_{i}}=\sum_{i=1}^{s} \beta_{i} \chi_{B_{i}}-\sum_{i=r+1}^{n} \alpha_{i} \chi_{A_{i}}
$$

Then $f^{\prime}$ is a rational function with positive integer coefficients; if

$$
\sum_{i=1}^{r} \alpha_{i} \mu\left(A_{i}\right)-\sum_{i=s+1}^{m} \beta_{i} \mu\left(B_{i}\right)=\sum_{i=1}^{s} \beta_{i} \mu\left(B_{i}\right)-\sum_{i=r+1}^{n} \alpha_{i} \mu\left(A_{i}\right)
$$

then

$$
\sum_{i=1}^{n} \alpha_{i} \mu\left(A_{i}\right)=\sum_{i=1}^{m} \beta_{i} \mu\left(B_{i}\right)
$$

THEOREM 5.11. If $G$ is a group without large paradoxical sets and

$$
f=\sum_{i=1}^{n} \alpha_{i} \chi_{A_{i}}=\sum_{i=1}^{m} \beta_{i} \chi_{B_{i}}
$$

is a rational function, where $A_{i}$ and $B_{i}$ are rational sets, then

$$
\sum_{i=1}^{n} \alpha_{i} \mu\left(A_{i}\right)=\sum_{i=1}^{m} \beta_{i} \mu\left(B_{i}\right)
$$

Proof. It follows from the preceding lemma that we may assume the $\alpha_{i}$ and $\beta_{i}$ are positive integers. Now choose

$$
s>\sum_{i=1}^{n} \alpha_{i}+\sum_{i=1}^{m} \beta_{i}
$$

and define the graph of $f, \operatorname{Gr}(f) \subset G \times C_{s}$, by

$$
\operatorname{Gr}(f)=\left\{(x, r) \in G \times C_{s}: 0<r \leqslant f(x)\right\} .
$$

Then by Lemma 5.8 there is a measure $m$ on the rational sets of $G \times C_{s}$ such that $m(A)=\mu(A)$ for rational subsets $A$ of $G$. We will show that

$$
m(\operatorname{Gr}(f))=\sum_{i=1}^{n} \alpha_{i} \mu\left(A_{i}\right)
$$

From the definition of $\operatorname{Gr}(f)$ it follows that we can define piecewise translations $h_{i j}$ for $i=1, \ldots, n$ and $j=1, \ldots, \alpha_{i}$ such that

$$
\operatorname{Gr}(f)=\bigcup_{i=1}^{n} \bigcup_{j=1}^{\alpha_{i}} h_{i j}\left(A_{i}\right)
$$


and $h_{i j}\left(A_{i}\right) \cap h_{i^{\prime} j^{\prime}}\left(A_{i^{\prime}}\right)=\varnothing$ unless $i=i^{\prime}$ and $j=j^{\prime}$. Then

$$
\begin{aligned}
m(\operatorname{Gr}(f)) & =\sum_{i=1}^{n} \sum_{j=1}^{\alpha_{i}} m\left(h_{i j}\left(A_{i}\right)\right)=\sum_{i=1}^{n} \sum_{j=1}^{\alpha_{i}} m\left(A_{i}\right) \\
& =\sum_{i=1}^{n} \alpha_{i} m\left(A_{i}\right)=\sum_{i=1}^{n} \alpha_{i} \mu\left(A_{i}\right) .
\end{aligned}
$$

The $h_{i j}\left(A_{i}\right)$ are rational in $G \times C_{s}$, since the $A_{i}$ are rational in $G$. If we now repeat the same argument for $f=\sum_{i=1}^{m} \beta_{i} \chi_{B_{i}}$, we get

$$
m(\operatorname{Gr}(f))=\sum_{i=1}^{m} \beta_{i} \mu\left(B_{i}\right) \text {. }
$$

Thus

$$
\sum_{i=1}^{n} \alpha_{i} \mu\left(A_{i}\right)=\sum_{i=1}^{m} \beta_{i} \mu\left(B_{i}\right)
$$

\section{Extension of the linear functional.}

Definition 6.1. If $f, g \in V$, then $f \approx g$ if

$$
f=\sum_{i=1}^{n} \alpha_{i} \chi_{A_{i}} \text { and } g=\sum_{i=1}^{n} \alpha_{i} \chi_{h_{i}\left(A_{i}\right)}+\sum_{j=1}^{s} \beta_{j} \chi_{B_{j}},
$$

where the $h_{i}$ are piecewise translations and the $B_{j}$ are rational sets with $\mu\left(B_{j}\right)=0$.

If $f, g \in V$, then $f \sim g$ if

$$
f=\sum_{i=1}^{n} \alpha_{i} \chi_{A_{i}} \text { and } g=\sum_{i=1}^{n} \alpha_{i} \chi_{h_{i}\left(A_{i}\right)}
$$

where the $h_{i}$ are piecewise translations.

It follows from the properties of piecewise translations that there are equivalence relations.

If $f_{1} \approx g_{1}$ and $f_{2} \approx g_{2}$, then it is clear that $f_{1}+f_{2} \approx g_{1}+g_{2}$, and $a f_{1} \approx a g_{1}$ for any constant $a$.

Similarly, if $f_{1} \sim g_{1}$ and $f_{2} \sim g_{2}$, then $f_{1}+f_{2} \sim g_{1}+g_{2}$, and $a f_{1} \sim a g_{1}$ for any constant $a$.

Let $V^{\prime}$ be the collection of equivalence classes with respect to $\approx$ of $V$. Then $V^{\prime}$ is a linear space.

THEOREM 6.2. If $f, g \in M$ and $f \approx g$, then $\lambda(f)=\lambda(g)$.

LEMMA 0. If $f=f_{1}+f_{2}$, where $f, f_{1}, f_{2}$ are finite linear combinations of characteristic functions and are positive integer-valued; and if $\operatorname{Gr}\left(f_{1}\right), \operatorname{Gr}\left(f_{2}\right)$ are rational sets in $G \times C_{s}$, then

$$
m(\operatorname{Gr}(f))=m\left(\operatorname{Gr}\left(f_{1}\right)\right)+m\left(\operatorname{Gr}\left(f_{2}\right)\right) \text {. }
$$


Proof. Since $f=f_{1}+f_{2}$, it follows from the definition of $\mathrm{Gr}$ that, for some piecewise translation $H$ of $G \times C_{s}$,

$$
\operatorname{Gr}(f)=\operatorname{Gr}\left(f_{1}\right) \cup H\left(\operatorname{Gr}\left(f_{2}\right)\right)
$$

and

$$
\operatorname{Gr}\left(f_{1}\right) \cap H\left(\operatorname{Gr}\left(f_{2}\right)\right)=\varnothing
$$

Then

$$
m(\operatorname{Gr}(f))=m\left(\operatorname{Gr}\left(f_{1}\right)\right)+m\left(H\left(\operatorname{Gr}\left(f_{2}\right)\right)\right)
$$

and

$$
m(\operatorname{Gr}(f))=m\left(\operatorname{Gr}\left(f_{1}\right)\right)+m\left(\operatorname{Gr}\left(f_{2}\right)\right) \text {. }
$$

LEMMA 1. If $f=\sum_{i=1}^{m} \alpha_{i} \chi_{A_{i}}=\sum_{i=1}^{p} \beta_{i} \chi_{B_{i}}$ and

$$
g=\sum_{i=1}^{m} \alpha_{i} \chi_{h_{i}\left(A_{i}\right)}=\sum_{i=1}^{r} \gamma_{i} \chi_{C_{i}}
$$

where the $\gamma_{i}, \beta_{i}$, are positive integers and the $\alpha_{i}$ are integers (not necessarily positive), and the $B_{i}$ and $C_{i}$ are rational sets, then $\lambda(f)=\lambda(g)$.

Proof. We proved in Theorem 5.11 that if $f=\sum_{i=1}^{p} \beta_{i} \chi_{B_{i}}$, where the $\beta_{i}$ are positive integers and the $B_{i}$ are rational sets, then

$$
\sum_{i=1}^{p} \beta_{i} \mu\left(B_{i}\right)=m \operatorname{Gr}(f),
$$

where

$$
\operatorname{Gr}(f)=\left\{(x, r) \in G \times C_{s}: 0<r \leqslant f(x)\right\},
$$

and $s$ can be any integer greater than $\max \{f(x)$ and $g(x)\}$; and $m$ is a finitely additive, piecewise-translation invariant set function defined on rational subsets of $G \times C_{s}$, which is identical with $\mu$ for rational subsets of $G$.

The existence of such a set function is proved in Lemma 5.8.

From Theorem 5.11 we also have

$$
\sum_{i=1}^{r} \gamma_{i} \mu\left(C_{i}\right)=m(\operatorname{Gr}(g))
$$

We will now show that $m(\operatorname{Gr}(f))=m(\operatorname{Gr}(g))$.

Define

$$
\begin{gathered}
f_{+}=\sum_{\alpha_{i}>0} \alpha_{i} \chi_{A_{i}} \text { and } f_{-}=\sum_{\alpha_{i}<0}\left(-\alpha_{i} \chi_{A_{i}}\right), \\
g_{+}=\sum_{\alpha_{i}>0} \alpha_{i} \chi_{h_{i}\left(A_{i}\right)} \text { and } g_{-}=\sum_{\alpha_{i}<0}\left(-\alpha_{i} \chi_{h_{i}\left(A_{i}\right)}\right) .
\end{gathered}
$$

Then $f=f_{+}-f_{-}$and $g=g_{+}-g_{-}$.

For each $i=1, \ldots, m$ such that $\alpha_{i}<0$, choose a rational set $A_{i}^{\prime} \subset G$ with 
$A_{i} \subset A_{i}^{\prime}$. Since $G$ is rational, such a set always exists. Then let

$$
f_{-}^{\prime}=\sum_{\alpha_{i}<0}\left(-\alpha_{i} \chi_{A_{i}}\right)+\sum_{\alpha_{i}<0}\left(-\alpha_{i} \chi_{\left(A_{i}^{\prime}-A_{i}\right)}\right)=\sum_{\alpha_{i}<0}\left(-\alpha_{i} \chi_{A_{i}^{\prime}}\right)
$$

and let

$$
f_{+}^{\prime}=f_{+}+\sum_{\alpha_{i}<0}\left(-\alpha_{i} \chi_{\left(A_{i}^{\prime}-A_{i}\right)}\right)=\sum_{\alpha_{i}>0}\left(\alpha_{i} \chi_{A_{i}}\right)+\sum_{\alpha_{i}<0}\left(-\alpha_{i} \chi_{\left(A_{i}^{\prime}-A_{i}\right)}\right) .
$$

Then $f=f_{+}^{\prime}-f_{-}^{\prime}$, and since $f$ and $f_{-}^{\prime}$ are rational functions, so is $f_{+}^{\prime}$.

Let

$$
g_{-}^{\prime}=g_{-}+\sum_{\alpha_{i}<0}\left(-\alpha_{i} \chi_{\left(A_{i}^{\prime}-A_{i}\right)}\right)
$$

and

$$
g_{+}^{\prime}=g_{+}+\sum_{\alpha_{i}<0}\left(-\alpha_{i} \chi_{\left(A_{i}^{\prime}-A_{i}\right)}\right)
$$

Then $g=g_{+}^{\prime}-g_{-}^{\prime}$, and $f_{+}^{\prime} \sim g_{+}^{\prime}$ and $f_{-}^{\prime} \sim g_{-}^{\prime}$.

It can be shown from the definition of $\sim$ that if $f_{1}, f_{2}$ are functions for which $\mathrm{Gr}$ is defined and $f_{1} \sim f_{2}$, then $\operatorname{Gr}\left(f_{1}\right)$ is a piecewise translation of $\operatorname{Gr}\left(f_{2}\right)$. Therefore, $\operatorname{Gr}\left(g_{+}^{\prime}\right)$ is a piecewise translation of $\operatorname{Gr}\left(f_{+}^{\prime}\right)$ and $\operatorname{Gr}\left(g_{-}^{\prime}\right)$ is a piecewise translation of $\operatorname{Gr}\left(f_{-}^{\prime}\right)$.

Thus $\operatorname{Gr}\left(g_{+}^{\prime}\right)$ and $\operatorname{Gr}\left(g_{-}^{\prime}\right)$ are rational sets in $G \times C_{s}$ since they are piecewise translations of $\operatorname{Gr}\left(f_{+}^{\prime}\right)$ and $\operatorname{Gr}\left(f_{-}^{\prime}\right)$.

Then $f_{+}^{\prime}=f+f_{-}^{\prime}$ and $g_{+}^{\prime}=g+g_{-}^{\prime}$.

By Lemma 0:

$$
m\left(\operatorname{Gr}\left(f_{+}^{\prime}\right)\right)=m(\operatorname{Gr}(f))+m\left(\operatorname{Gr}\left(f_{-}^{\prime}\right)\right)
$$

and

$$
m\left(\operatorname{Gr}\left(g_{+}^{\prime}\right)\right)=m(\operatorname{Gr}(g))+m\left(\operatorname{Gr}\left(g_{-}^{\prime}\right)\right) .
$$

Since $\operatorname{Gr}\left(f_{+}^{\prime}\right)$ and $\operatorname{Gr}\left(f_{-}^{\prime}\right)$ are piecewise translations of $\operatorname{Gr}\left(g_{+}^{\prime}\right)$ and $\operatorname{Gr}\left(g_{-}^{\prime}\right)$,

$$
m\left(\operatorname{Gr}\left(f_{+}^{\prime}\right)\right)=m\left(\operatorname{Gr}\left(g_{+}^{\prime}\right)\right) \text { and } m\left(\operatorname{Gr}\left(f_{-}^{\prime}\right)\right)=m\left(\operatorname{Gr}\left(g_{-}^{\prime}\right)\right)
$$

Thus

$$
m(\operatorname{Gr}(f))=m(\operatorname{Gr}(g)) \text { and } \lambda(f)=\lambda(g) .
$$

LEMMA 2. If $f=\sum_{i=1}^{m} \alpha_{i} \chi_{A_{i}}=\sum_{i=1}^{p} \beta_{i} \chi_{B_{i}}$ and $g=\sum_{i=1}^{m} \alpha_{i} \chi_{h_{i}\left(A_{i}\right)}=$ $\sum_{i=1}^{r} \gamma_{i} \chi_{C_{i}}$, where the $\alpha_{i}, \beta_{i}, \gamma_{i}$ are integers, and the $B_{i}$ and $C_{i}$ are rational sets, then $\lambda(f)=\lambda(g)$.

Proof. Let

$$
f^{+}=\sum_{\beta_{i}>0} \beta_{i} \chi_{B_{i}} \text { and } f^{-}=\sum_{\beta_{i}<0}-\beta_{i} \chi_{B_{i}}
$$


and

$$
g^{+}=\sum_{\gamma_{i}>0} \gamma_{i} \chi_{C_{i}} \text { and } g^{-}=\sum_{\gamma_{i}<0}-\gamma_{i} \chi_{C_{i}}
$$

Then $f=f^{+}-f^{-}$and $g=g^{+}-g^{-}$.

Thus $f^{+}, f^{-}, g^{+}$, and $g^{-}$are rational functions, and $f^{+}-f^{-} \sim g^{+}-g^{-}$. Then $f^{+}+g^{-} \sim g^{+}+f^{-}$. These are rational functions with positive integer coefficients, and it follows from Lemma 1 that $\lambda\left(f^{+}+g^{-}\right)=\lambda\left(g^{+}+f^{-}\right)$. Then, since $\lambda$ is linear, we have $\lambda\left(f^{+}\right)+\lambda\left(g^{-}\right)=\lambda\left(g^{+}\right)+\lambda\left(f^{-}\right)$and $\lambda\left(f^{+}\right)$ $-\lambda\left(f^{-}\right)=\lambda\left(g^{+}\right)-\lambda\left(g^{-}\right)$or $\lambda(f)=\lambda(g)$.

LEMMA 3. If $f=\sum_{i=1}^{m} \alpha_{i} \chi_{A_{i}}=\sum_{i=1}^{p} \beta_{i} \chi_{B_{i}}$ and $g=\sum_{i=1}^{m} \alpha_{i} \chi_{h_{i}\left(A_{i}\right)}=$ $\sum_{i=1}^{r} \gamma_{i} \chi_{C_{i}}$, where the $\alpha_{i}, \beta_{i}, \gamma_{i}$ are rational numbers and $B_{i}$ and $C_{i}$ are rational sets, then $\lambda(f)=\lambda(g)$.

Proof. If $D=$ L.C.D. $\left(\alpha_{i}, \beta_{i}, \gamma_{i}\right)$, then $D f$ and $D g$ will satisfy the hypotheses of Lemma 2 and $\lambda(D f)=\lambda(D g)$. Then $D \lambda(f)=D \lambda(g)$ and $\lambda(f)=\lambda(g)$.

LEMMA 4. If $f, g \in M$ and $f \sim g$, then $\lambda(f)=\lambda(g)$.

Proof. Suppose $f=\sum_{i=1}^{m} \alpha_{i} \chi_{A_{i}}=\sum_{i=1}^{s} \beta_{i} \chi_{B_{i}}$ and $g=\sum_{i=1}^{s} \alpha_{i} \chi_{h_{i}\left(A_{i}\right)}=$ $\sum_{i=1}^{r} \gamma_{i} \chi_{C_{i}}$, where $\alpha_{i}, \beta_{i}, \gamma_{i} \in \mathbf{R}$ and $B_{i}$ and $C_{i}$ are rational sets.

Let $C$ be a Hamel basis of $\mathbf{R}$ over $\mathbf{Q}$. Then for each $\alpha_{i}, \beta_{i}, \gamma_{i}$ there is a unique function $\alpha_{i}(c), \beta_{i}(c), \gamma_{i}(c)$ from $C \rightarrow \mathbf{Q}$ which equals 0 except for finitely many $c$ 's such that

$$
\alpha_{i}=\sum_{c \in C} \alpha_{i}(c) \cdot c, \quad \beta_{i}=\sum_{c \in C} \beta_{i}(c) \cdot c, \quad \gamma_{i}=\sum_{c \in C} \gamma_{i}(c) \cdot c .
$$

Let $c_{1}, c_{2}, \ldots, c_{n}$ be the finitely many elements of $C$ such that $\alpha_{i}(c) \neq 0$ or $\beta_{i}(c) \neq 0$ or $\gamma_{i}(c) \neq 0$, for some $i$.

Then

$$
\alpha_{i}=\sum_{j=1}^{n} \alpha_{i}\left(c_{j}\right) \cdot c_{j}, \quad \beta_{i}=\sum_{j=1}^{n} \beta_{i}\left(c_{j}\right) \cdot c_{j}, \quad \gamma_{i}=\sum_{j=1}^{n} \gamma_{i}\left(c_{j}\right) \cdot c_{j} .
$$

Thus

$$
f=\sum_{i=1}^{m}\left(\sum_{j=1}^{n} \alpha_{i}\left(c_{j}\right) \cdot c_{j}\right) \chi_{A_{i}}=\sum_{i=1}^{s}\left(\sum_{j=1}^{n} \beta_{i}\left(c_{j}\right) \cdot c_{j}\right) \chi_{B_{i}}
$$

and

$$
g=\sum_{i=1}^{m}\left(\sum_{j=1}^{n} \alpha_{i}\left(c_{j}\right) \cdot c_{j}\right) \chi_{h_{i}\left(A_{i}\right)}=\sum_{i=1}^{r}\left(\sum_{j=1}^{n} \gamma_{i}\left(c_{j}\right) \cdot c_{j}\right) \chi_{c_{i}}
$$

Then

$$
\sum_{j=1}^{n}\left(\sum_{i=1}^{m} \alpha_{i}\left(c_{j}\right) \chi_{A_{i}}\right) c_{j}=\sum_{j=1}^{n}\left(\sum_{i=1}^{s} \beta_{i}\left(c_{j}\right) \chi_{B_{i}}\right) c_{j}
$$


and

$$
\sum_{j=1}^{n}\left(\sum_{i=1}^{m} \alpha_{i}\left(c_{j}\right) \chi_{h_{i}\left(A_{i}\right)}\right) c_{j}=\sum_{j=1}^{n}\left(\sum_{i=1}^{r} \gamma_{i}\left(c_{j}\right) \chi_{C_{i}}\right) c_{j}
$$

It follows from the linear independence of $C$ that for each $j=1,2, \ldots, n$

$$
\sum_{i=1}^{m} \alpha_{i}\left(c_{j}\right) \chi_{A_{i}}=\sum_{i=1}^{s} \beta_{i}\left(c_{j}\right) \chi_{B_{i}}
$$

and

$$
\sum_{i=1}^{m} \alpha_{i}\left(c_{j}\right) \chi_{h_{i}\left(A_{i}\right)}=\sum_{i=1}^{r} \gamma_{i}\left(c_{j}\right) \chi_{C_{i}}
$$

Let

$$
f_{j}=\sum_{i=1}^{m} \alpha_{i}\left(c_{j}\right) \chi_{A_{i}} \text { and } g_{j}=\sum_{i=1}^{m} \alpha_{i}\left(c_{j}\right) \chi_{h_{i}\left(A_{i}\right)} .
$$

Then $f_{j} \sim g_{j}$, and the coefficients $\alpha_{i}\left(c_{j}\right), \beta_{i}\left(c_{j}\right)$, and $\gamma_{i}\left(c_{j}\right)$ are rational. Further, equations (1) and (2) show that $f_{j}$ and $g_{j}$ are rational functions.

Thus by Lemma 3 we have $\lambda\left(f_{j}\right)=\lambda\left(g_{j}\right)$ for all $j=1,2, \ldots, n$. Then since $\lambda$ is linear we have

$$
\lambda f=\lambda\left(\sum_{j=1}^{n} c_{j} f_{j}\right)=\sum_{j=1}^{n} c_{j} \lambda\left(f_{j}\right)=\sum_{j=1}^{n} c_{j} \lambda\left(g_{j}\right)=\lambda\left(\sum_{j=1}^{n} c_{j} g_{j}\right)=\lambda g .
$$

This completes the proof of Lemmas 1-4.

Now suppose that

$$
f=\sum_{i=1}^{m} \alpha_{i} \chi_{A_{i}}=\sum_{i=1}^{s} \beta_{i} \chi_{B_{i}}
$$

and

$$
g=\sum_{i=1}^{m} \alpha_{i} \chi_{h_{i}\left(A_{i}\right)}+\sum_{i=1}^{t} \varphi_{i} \chi_{D_{i}}=\sum_{i=1}^{r} \gamma_{i} \chi_{C_{i}},
$$

where the $\alpha_{i}, \beta_{i}, \gamma_{i}, \varphi_{i} \in \mathbf{R}$, the $B_{i}, C_{i}, D_{i}$ are rational sets and $\mu\left(D_{i}\right)=0$.

Then let $g^{\prime}=g-\sum_{i=1}^{t} \varphi_{i} \chi_{D_{i}}$. By Lemma 4, $\lambda\left(g^{\prime}\right)=\lambda(f)$. But

$$
\lambda\left(g^{\prime}\right)=\lambda g-\lambda\left(\sum_{i=1}^{t} \varphi_{i} \chi_{D_{i}}\right)=\lambda g-\sum_{i=1}^{t} \varphi_{i} \mu\left(D_{i}\right)=\lambda g .
$$

Thus $\lambda(g)=\lambda(f)$. This completes the proof.

Definition 6.3. Define the relation $>$ on $V^{\prime}$ by defining $F>0$ if there exists $f \in F$ such that $f \geqslant 0$. Then $F_{1}>F_{2}$ if $F_{1}-F_{2}>0$.

THEOREM 6.4. If $f \geqslant 0$ and $g \leqslant 0$ and $f \sim g$, then $f=g=0$.

If $f>0$ and $g \leqslant 0$ and $f \approx g$, then $f-g=0$ for all $x \in G-E$, where $\mu(E)=0$. 
Proof. Suppose that

$$
f=\sum_{i=1}^{n} \alpha_{i} \chi_{A_{i}} \geqslant 0 \text { and } g=\sum_{i=1}^{n} \alpha_{i} \chi_{h_{i}\left(A_{i}\right)} \leqslant 0,
$$

where the $\alpha_{i}$ are integers.

Then

$$
f-g=\sum_{i=1}^{n} \alpha_{i} \chi_{A_{i}}-\sum_{i=1}^{n} \alpha_{i} \chi_{h_{i}\left(A_{i}\right)} \geqslant 0 .
$$

(We first prove this theorem for integer coefficients, and then extend it to functions with rational and real coefficients.) Then $f-g>0$ and $f-g \sim 0$. Since $f-g \geqslant 0$ and has positive integer values, we can define

$$
\operatorname{Gr}(f-g)=\left\{(x, r) \in G \times C_{s}: 0<(f-g)(x)<r\right\} \subset G \times C_{s} .
$$

Since $(f-g) \sim 0$ there exists a piecewise translation $H$ in $G \times C_{s}$ such that $H(\operatorname{Gr}(f-g))=\operatorname{Gr}(0)=\varnothing$. Then $\operatorname{Gr}(f-g)=\varnothing$. This implies that $f-g=$ 0 .

If for some $x \in G,(f-g)(x) \neq 0$, then $(f-g)(x)>0$ and $(x,(f-$ $g)(x)) \in \operatorname{Gr}(f-g)$. Thus $(f-g)=0$, and since $f>0$ and $-g>0$, it follows that $f=0=g$ for all $x \in G$.

Now let us suppose that $f=\sum_{i=1}^{n} \alpha_{i} \chi_{A_{i}}>0$ and $g=\sum_{i=1}^{n} \alpha_{i} \chi_{h_{i}\left(A_{i}\right)} \leqslant 0$, where the $\alpha_{i}$ 's are rational numbers. Let $D=$ L.C.D. $\left\{\alpha_{i}\right\}$. Then $D f$ and $D g$ satisfy the hypothesis of the case proven above and $D f=D g=0$. It follows that $f=g=0$.

Next suppose that $f=\sum_{i=1}^{n} \alpha_{i} \chi_{A_{i}} \geqslant 0$ and $g=\sum_{i=1}^{n} \alpha_{i} \chi_{h_{i}\left(A_{i}\right)} \leqslant 0$, where $\alpha_{i} \in \mathbf{R}$.

Choose a Hamel basis $C$ of $\mathbf{R}$ over the rationals. Then each $\alpha_{i}=$ $\Sigma_{c \in C} \alpha_{i}(c) \cdot c$, where $\alpha_{i}(c)$ is rational. Let $\left\{c_{j}: j=1, \ldots, m\right\}$ be the finite subset of $C$ such that $\alpha_{i}\left(c_{j}\right) \neq 0$ for some $i=1,2, \ldots, n$. Then $\alpha_{i}=$ $\sum_{j=1}^{m} \alpha_{i}\left(c_{j}\right) \cdot c_{j}$, where $\alpha_{i}\left(c_{j}\right)$ is rational. Thus

$$
f=\sum_{i=1}^{n} \alpha_{i} \chi_{A_{i}}=\sum_{i=1}^{n} \sum_{j=1}^{m} \alpha_{i}\left(c_{j}\right) \cdot c_{j} \chi_{A_{i}}>0
$$

and

$$
g=\sum_{i=1}^{n} \alpha_{i} \chi_{h_{i}\left(A_{i}\right)}=\sum_{i=1}^{n} \sum_{j=1}^{m} \alpha_{i}\left(c_{j}\right) \cdot c_{j} \chi_{h_{i}\left(A_{i}\right)}<0
$$

Let

$$
f_{j}=\sum_{i=1}^{n} \alpha_{i}\left(c_{j}\right) \chi_{A_{i}} \text { and } g_{j}=\sum_{i=1}^{n} \alpha_{i}\left(c_{j}\right) \chi_{h_{i}\left(A_{i}\right)}
$$


Then

$$
f=\sum_{j=1}^{m} c_{j} f_{j} \text { and } g=\sum_{j=1}^{m} c_{j} g_{j}
$$

Choose $m$ monotonic decreasing sequences of rationals $\alpha_{j k}$ for $j=$ $1,2, \ldots, m$ such that $\lim _{k \rightarrow \infty} \alpha_{j k}=c_{j}$, for each $j$. Also choose $m$ monotonic increasing sequences of rationals $\beta_{j k}$ for $j=1,2, \ldots, m$ such that $\lim _{k \rightarrow \infty} \beta_{j k}$ $=c_{j}$.

Let

$$
\bar{f}_{k}=\sum_{j=1}^{m} \alpha_{j k} f_{j}, \quad \bar{g}_{k}=\sum_{j=1}^{m} \alpha_{j k} g_{j}
$$

and

$$
\underline{f}_{k}=\sum_{j=1}^{m} \beta_{j k} f_{j}, \quad \underline{g}_{k}=\sum_{j=1}^{m} \beta_{j k} g_{j} .
$$

Then $\bar{f}_{k} \geqslant f \geqslant f_{k}$ and $\bar{g}_{k} \geqslant g \geqslant g_{k}$ for every $k$.

Let $\varepsilon_{1}$ be the smallest nonzero value of $f$ and let $\varepsilon_{2}$ be the absolute value of the largest nonzero value of $g$. Let $\varepsilon=\min \left\{\varepsilon_{1}, \varepsilon_{2}\right\}$, and choose $k_{0}$ such that $\left|\alpha_{k j}-c_{j}\right|<\varepsilon / m M$ and $\left|\beta_{k j}-c_{j}\right|<\varepsilon / m M$ for all $j=1,2, \ldots, m$, for all $k>k_{0}$, and where $M=\max \left\{f_{j}, g_{j}: j=1, \ldots, m\right\}$. We know that $\bar{f}_{k}>f>0$ and $g_{k}<g<0$ are true for all $k$.

For sufficiently large $k$ we have $0 \leqslant f-f_{k}<\varepsilon$ and $0<\bar{g}_{k}-g<\varepsilon$.

If $f(x)>0$, then $f(x) \geqslant \varepsilon$ and $f_{k}(x) \geqslant 0$. If $f(x)=0$, then since $f=$ $\sum_{j=1}^{m} c_{j} f_{j}$, it is a consequence of the linear independence of the Hamel basis that $f_{j}(x)=0$ for all $j=1,2, \ldots, m$. But then

$$
f_{k}(x)=\sum_{j=1}^{m} \beta_{j k} f_{j}(x)=0 .
$$

Thus $f_{k}>0$ for all $k \geqslant k_{0}$.

Similarly, if $g(x)<0$, then $g(x)<-\varepsilon$ and $\bar{g}_{k}(x)<0$. If $g(x)=0$, then $g_{j}(x)=0$ for all $j$ and

$$
\bar{g}_{k}(x)=\sum_{j=1}^{m} \alpha_{j k} g_{j}(x)=0 .
$$

Thus $\bar{g}_{k}(x)<0$ for $k \geqslant k_{0}$.

It is easy to see from the definitions

$$
f_{j}=\sum_{i=1}^{n} \alpha_{i}\left(c_{j}\right) \chi_{A_{i}} \text { and } g_{j}=\sum_{i=1}^{n} \alpha_{i}\left(c_{j}\right) \chi_{h_{i}\left(A_{i}\right)}
$$

that $f_{j} \sim g_{j}$ for each $j=1,2, \ldots, m$. It also follows from the definitions of $\bar{f}_{k}$, $\bar{g}_{k}, f_{k}$, and $g_{k}$ as linear combinations of the $f_{j}$ and $g_{j}$ that $\bar{f}_{k} \sim \bar{g}_{k}$ and $f_{k} \sim g_{k}$. Since these functions are linear combinations with rational coefficients of 
characteristic functions, the results of the preceding section of the proof apply and it follows that $\bar{f}_{k}=\bar{g}_{k}=0$ and $f_{k}=g_{k}=0$. Then $f=g=0$.

This proves the first part of the theorem.

Now if

$$
f=\sum_{i=1}^{n} \alpha_{i} \chi_{A_{i}} \geqslant 0 \text { and } g=\sum_{i=1}^{n} \alpha_{i} \chi_{h_{i}\left(A_{i}\right)}+\sum_{i=1}^{m} \beta_{i} \chi_{B_{i}}
$$

where $\alpha_{i}, \beta_{i} \in \mathbf{R}$ and the $B_{i}$ are rational sets with $\mu\left(B_{i}\right)=0$, we define $f^{\prime}$ and $g^{\prime}$ by $f^{\prime}(x)=f(x)$ and $g^{\prime}(x)=g(x)$ if $x \in G-\cup_{i=1}^{m} B_{i}$; and $f(x)=0$ and $g(x)=0$ if $x \in \cup_{i=1}^{m} B_{i}$. Then $f^{\prime} \geqslant 0$ and $g^{\prime} \leqslant 0$ and $f^{\prime} \sim g^{\prime}$. Thus $f^{\prime}=g^{\prime}$ $=0$, and $f=g=0$ for all $x \notin \cup_{i=1}^{m} B_{i}$ and $\mu\left(\cup_{i=1}^{m} B_{i}\right)=0$.

It follows immediately from Theorem 6.4 that if $f \geqslant 0$ and $g \leqslant 0$ and $f \approx g$, then $f \approx 0 \approx g$.

LEMMA 6.5. The relation $\geqslant$ is a partial ordering of $V^{\prime}$.

Proof. (1) Reflexive. It is clear that $F \geqslant F$.

(2) Transitive. If $F_{1} \geqslant F_{2}$ and $F_{2} \geqslant F_{3}$, then $F_{1}-F_{2} \geqslant 0$ and $F_{2}-F_{3} \geqslant 0$. Then there exist $f \in F_{1}-F_{2}$ and $g \in F_{2}-F_{3}$ such that $f \geqslant 0$ and $g \geqslant 0$. Then $f+g \in\left(F_{1}-F_{2}\right)+\left(F_{2}-F_{3}\right)=F_{1}-F_{3}$ and $f+g>0$. Thus $F_{1} \geqslant$ $F_{3}$.

(3) Antisymmetric. If $F_{1} \geqslant F_{2}$ and $F_{2} \geqslant F_{1}$, then there exist $f \in F_{1}-F_{2}$ and $g \in F_{2}-F_{1}$ such that $f \geqslant 0$ and $g \geqslant 0$. Then $-g \in F_{1}-F_{2}$ and $-g<0$. Thus $f \approx(-g)$. Then it follows from Lemma 6.3 that $f \approx 0$, and thus $F_{1}=F_{2}$.

Definition 6.6. Let $M^{\prime}=\left\{F \in V^{\prime}: F \cap M \neq \varnothing\right\}$. Then $M^{\prime}$ is a linear space. If $f_{1} \in M \cap F_{1}$ and $f_{2} \in M \cap F_{2}$, then $f_{1}+f_{2} \in M \cap\left(F_{1}+F_{2}\right)$ and $a f_{1} \in M \cap a F_{1}$ for any constant $a$. Now we define a linear functional on $M^{\prime}$ by $L(F)=\lambda(f)$ for any $f \in F \cap M$. Then $L$ is a nonnegative functional since $\lambda$ is.

The next theorem, here for convenience, is adapted from Theorem 14 of Banach [1].

THEOREM 6.7. If a nonnegative linear functional $L_{\beta}$ is defined on the linear space $V_{\beta} \subset V^{\prime}$ and $\varphi \in V^{\prime}-V_{\beta}$ with $F_{1} \geqslant \varphi \geqslant F_{2}, F_{1}$ and $F_{2} \in V_{\beta}$, then $L_{\beta}$ can be extended to a nonnegative linear functional $L_{\beta+1}$ defined on $V_{\beta+1}=$ $\left(V_{\beta}, \varphi\right)$ such that $L_{\beta+1}(F)=L_{\beta}(F)$ if $F \in V_{\beta}$.

Proof. Let $\alpha=\inf \left\{L_{\beta}(F): F \in V_{\beta}\right.$ and $\left.F \geqslant \varphi\right\}$. Every coset in $V_{\beta+1}$ is of the form $F+c \varphi$. We can define $L_{\beta+1}(F+c \varphi)=L_{\beta}(F)+c \alpha$. It is clear that this is well-defined and linear. It remains to show that $L_{\beta+1}$ is nonnegative.

Suppose $F+c \varphi \geqslant 0$. If $c=0$, then $L_{\beta+1}(F+0 \cdot \varphi)-L_{\beta}(F) \geqslant 0$, since $L_{\beta}$ is nonnegative. 
If $c>0$, then $\varphi \geqslant-F / c$ and for every $F_{1} \geqslant 0$ we have $F_{1} \geqslant-F / c$ or $F_{1}+F / c \geqslant 0$. Thus $L_{\beta}\left(F_{1}+F / c\right) \geqslant 0$ and $c \alpha+L_{\beta}(F) \geqslant 0$.

If $c<0$, then $F /-c-\varphi \geqslant 0$. Thus $L_{\beta}(-F / c)>\alpha$ or $-L_{\beta}(F) / c \geqslant \alpha$, from which follows $L_{\beta}(F)+c \alpha \geqslant 0$, since $c<0$.

Thus $L_{\beta+1}$ is a nonnegative linear functional on $V_{\beta+1}$.

TheOREM 6.8. The nonnegative linear functional $L$ defined on $M^{\prime}$ can be extended to a nonnegative linear functional $L^{\prime}$ defined on $V^{\prime}$ such that if $F \in M^{\prime}$ then $L^{\prime}(F)=L(F)$.

Proof. See Banach [1, Theorems 15 and 16].

Since every $f \in V$ is bounded and since all constant functions are in $M$, we know that for every $\varphi \in V^{\prime}$ there are $F_{1}, F_{2} \in M$ such that $F_{1} \geqslant \varphi \geqslant F_{2}$. Thus we can apply the preceding theorem and transfinite induction to show that $L$ can be extended to a nonnegative linear functional $L^{\prime}$ on $V^{\prime}$.

It is now possible to define a nonnegative linear functional $\lambda^{\prime}$ on $V$ such that if $f \in M$, then $\lambda^{\prime}(f)=\lambda(f)$. We define $\lambda^{\prime}(f)=L^{\prime}(F)$, where $f \in F$.

Then $\lambda^{\prime}$ is a translation invariant nonnegative linear functional on $V$, i.e., $\lambda^{\prime}$ satisfies the following conditions:

(1) $\lambda^{\prime}\left(x_{G}\right)=1$,

(2) $\lambda^{\prime}(a f+b g)=a \lambda^{\prime}(f)+b \lambda^{\prime}(g)$,

(3) $\lambda^{\prime}(f) \geqslant 0$ if $f \geqslant 0$,

(4) $\lambda^{\prime}\left(\sum_{i=1}^{n} \alpha_{i} \chi_{h_{i}\left(A_{i}\right)}\right)=\lambda^{\prime}\left(\sum_{i=1}^{n} \alpha_{i} \chi_{A_{i}}\right)$, if the $h_{i}$ are piecewise translations.

We can now easily define a Banach measure on $G$ by defining $\mu(A)=$ $\lambda^{\prime}\left(\chi_{A}\right)$ for all $A \subset G$. Then it is obvious that $\mu$ satisfies the conditions:

(1) $\mu(G)=1$,

(2) $\mu(A \cup B)=\mu(A)+\mu(B)$, if $A \cap B=\varnothing$,

(3) $\mu(h(A))=\mu(A)$ if $h$ is a piecewise translation,

(4) $\mu(A) \geqslant 0$ for all $A \subset G$.

Since the piecewise translation $h$ in Condition 3 is a left piecewise translation, $\mu$ is a left translation invariant measure and $G$ is left amenable. It is well known (see Day [5] and Greenleaf [6]) that if a group $G$ is left amenable, then $G$ is right amenable, and that if $G$ is left and right amenable, then $G$ is amenable.

This completes the proof of our main result.

THEOREM 6.9. A group is amenable if and only if it does not contain a large paradoxical set.

7. Comments and conjectures. The proof of Theorem 6.9 relies heavily on the use of $G \times C_{n}$ that is permitted by the definition of "large paradoxical set" as no large 2-into-1 in any $G \times C_{n}$. Clearly the theorem would be more satisfying if we could alter the definition of " $G$ contains no large paradoxical 
sets" to " $G$ contains no large 2-into-1" and avoid mention of the direct product $G \times C_{n}$. This would also be a more natural generalization of Banach and Tarski's paradoxical sets. To do this and still have Theorem 6.9 be true it would be sufficient to prove the following conjecture:

CONJECTURE 7.1. Given a group $G$ and a finite cyclic group $C_{n}, G$ contains a large 2-into-1 if and only if $G \times C_{n}$ does.

To prove this, it suffices to show:

Conjecture $7.1^{\prime}$. If $G \times C_{2}$ contains a large 2 -into-1, then so does $G$.

If it is true for $C_{2}$ then it is true for any $C_{n}$. The converse of Conjecture 7.1' is obvious.

The following conjectures are of considerable interest and closely related to this topic:

CONJECTURE 7.2. A group is amenable if and only if it does not contain a noncyclic free subgroup.

CONJECTURE 7.3. A group contains a large 2-into- 1 if and only if it contains a noncyclic free subgroup.

In each of these conjectures one direction is easy. Thus we can rewrite them to show only the unknown direction:

CONJECTURE 7.2'. If a group is not amenable then it contains a noncyclic free subgroup.

CONJECTURE $7.3^{\prime}$. If a group contains a large 2-into-1 then it contains a noncyclic free subgroup.

Since the converse of Conjecture 7.3' is not trivial we include the proof.

THEOREM 7.4. If a group contains a noncyclic free group, then it contains a large 2-into-1.

Proof. Let $F \doteq\langle a, b\rangle \subset G$. Then $G=\cup_{g \in E} F g$. Elements of $F$ can be written as words in $a$ and $b, w=a^{n_{1}} b^{n_{2}} a^{n_{3}} \ldots b^{n_{k}}$. Define piecewise translations $\psi$ and $\varphi$ as follows: if $w$ begins in a negative power of $a$, then $\varphi w=a^{-1} w$; otherwise $\varphi w=a w$. If $w$ begins in a negative power of $b$, then $\psi w=b^{-1} w$; otherwise $\psi w=b w$. Then $\varphi F \cap \psi F=\varnothing$, since every word of $\varphi F$ begins in $a$ or $a^{-1}$ and every word of $\psi F$ begins in $b$ or $b^{-1}$. Then, since $G=\cup_{g \in E} F g$, for each $x \in G$ we can write $x=w g$, where $w \in F$, and we can define $\varphi x$ and $\psi x$ for each $x \in G$ by $\varphi x=\varphi w g$ and $\psi x=\psi w g$. Then, since $\varphi F \subset F$ and $\psi F \subset F$ and $\varphi F \cap \psi F=\varnothing$, it is clear that $\varphi G \cap \psi G=\varnothing$. Thus since $\varphi G \cup \psi G \subset G, G$ is a large 2-into-1.

The conjectures $7.2^{\prime}$ and $7.3^{\prime}$ are closely related.

THEOREM 7.5. Conjecture 7.2' implies and is implied by Conjecture 7.3'.

Proof. Suppose $G$ is not amenable. Then by Theorem 6.9, $G$ contains a large paradoxical set. Thus $G \times C_{n}$ contains a large 2-into-1. Then according 
to Conjecture 7.3', $G \times C_{n}$ contains a noncyclic free subgroup. But it is easy to see that if $G \times C_{n}$ contains a noncyclic free group then so does $G$. The other direction is obvious.

To show that there is in fact a distinction between groups which contain a paradoxical set and groups which contain a large paradoxical set, we present an example of a group which contains no large paradoxical set but does contain a 2 -into-1. Let $G$ be the group of isometries of the plane. Then $G$ is amenable. The group $K$ of translations of the plane contains the commutator subgroup of $G$. So $G / K$ and $K$ are abelian and therefore amenable. Thus by a theorem of von Neumann [3], $G$ is amenable. It can be shown that $G$ contains a free semigroup on two generators. We now quote Theorem 1.7 from Sherman [9]: the group $G$ contains a 2-into-1 by translation if and only if $G$ contains a free semigroup on two generators.

Thus there is a set $S \subset G$ such that $a S \cup b S \subset S$ and $a S \cap b S=\varnothing$ for some $a, b \in G$. Since the translations $a, b$ are also piecewise translations, $S$ is a 2-into-1.

Hence $G$ is an amenable group which contains a 2-into-1. But from Theorem 6.9 we know it cannot contain a large 2-into-1. Therefore $G$ contains a paradoxical set but no large paradoxical set.

\section{BIBLIOGRAPHY}

1. Stefan Banach, Sur le problème de la mésure, Fund. Math. 4 (1923), 7-31.

2. John von Neumann, Zur allegemeinen Theorie des Masses, Fund. Math. 13 (1929), 73-116.

3. Felix Hausdorff, Grundzüge der Mengenlehre, Chelsea, New York, 1914.

4. S. Banach and A. Tarski, Sur la décomposition des ensembles de pointes en parties respectivement congruentes, Fund. Math. 6 (1924), 244-277.

5. M. Day, Amenable semigroups, Illinois J. Math. 1 (1957), 509-544.

6. F. P. Greenleaf, Invariant means on topological groups and their applications, Van Nostrand Math. Studies, no. 16, Van Nostrand Reinhold, New York, 1969.

7. Erling Folner, Generalization of a theorem of Bogoliouboff to topological abelian groups with an appendix on Banach mean values in non-abelian groups, Math. Scand. 2 (1954), 5-18.

8. On groups with full Banach mean value, Math. Scand. 3 (1955), 243-254.

9. Jon Sherman, Paradoxical sets and amenability in groups, Doctoral dissertation, UCLA, 1975.

Department of Mathematics, University of California, los Angeles, California 90024

Current address: 11736 Woodbine Avenue, Los Angeles, California 90066 